\title{
Performance Analysis of Reed-Solomon Codes for Effective Use in Survivable Wireless Sensor Networks
}

\author{
Ruiping Ma \\ Department of Electrical and Computer Engineering, \\ University of Massachusetts, Dartmouth, MA, USA. \\ E-mail: ruiping2008@gmail.com

\section{Liudong Xing} \\ Department of Electrical and Computer Engineering, \\ University of Massachusetts, Dartmouth, MA, USA. \\ Corresponding author: liudong.xing@umassd.edu

\section{Yujie Wang} \\ School of Mechanical and Electrical Engineering, \\ University of Electronic Science and Technology of China, Chengdu, China. \\ E-mail: yujie.wang@uestc.edu.cn \\ (Received October 14, 2019; Accepted October 25, 2019)
}

\begin{abstract}
In wireless sensor networks, data transmitted over wireless channels can get corrupted or lost due to channel noises and interferences. Error control coding is a technique that enables reliable delivery of digital data over unreliable communication channels. By adding redundancy to the transmitted data, error control codes allow the decoder to recover the original data based on partial data information received. The codes allow the receiver to recover errors or data loss without retransmission, thus making them suitable for applications where retransmissions are costly or impossible. Due to the stringent energy constraint of battery-powered sensor nodes, it is vital to use the energy-efficient error control scheme. In this paper, we evaluate the performance of Reed-Solomon (RS) codes with the objective to optimize their design factors, leading to optimal bit error performance over both Additive White Gaussian Noise (AWGN) channels and Rayleigh fading channels.
\end{abstract}

Keywords- Error correction code, Bit error rate, Signal-to-noise ratio, AWGN channel, Rayleigh fading channel, Performance analysis.

\section{Introduction}

Data transmitted over wireless sensor networks (WSNs) are vulnerable to errors and corruption due to channel noises, interferences, and malicious attacks (Zonouz et al., 2015; Wang et al., 2017). Typically, encryption can be performed to protect the data from the malicious attacks, but the secured data by encryption can be more sensitive to transmission errors (Ma et al., 2011). It is important to provide a proper error control scheme to reduce the bit error rate (BER). Due to the stringent energy constraint of battery-powered sensor nodes, it is vital to use energy-efficient error control schemes to efficiently recover the lost or erroneous information (original or encrypted) to save the retransmission overhead (Ma et al., 2006; Ma et al., 2007; Ma et al., 2009; Ma et al., 2012).

In digital communications, error correcting codes (ECCs) or forward error correction (FEC) codes have been traditionally used to control errors in data transmissions over unreliable or noisy 
International Journal of Mathematical, Engineering and Management Sciences

Vol. 5, No. 1, 13-28, 2020

https://doi.org/10.33889/IJMEMS.2020.5.1.002

communication channels (Abedi, 2011; Islam and Han, 2011; Alrajeh et al., 2015). By introducing redundancy into the transmitted data, ECCs provide capability for the decoder to detect and even correct errors happening to the data without retransmissions. The use of ECCs over a noisy channel can provide better BER performance for the same signal-to-noise ratio (SNR) compared to an un-coded system, or can provide the same BER at a lower SNR than the un-coded system (Howard et al., 2006). The coding gain achieved by using error-correction coding results in transmitter energy savings, but at the cost of complexity and energy overhead of the redundant parity bits and of the encoding/decoding computation. Usually there is a tradeoff between the coding gain and the overhead of the code.

As one type of the FEC codes, Reed-Solomon (RS) codes have been widely used to withstand the effects of channel errors thus to improve communication performance in traditional communication systems (Bettayeb et al., 2019; Yigit et al., 2019). Due to the energy-constraint of sensor nodes, it is crucial that we evaluate the performance of RS codes and choose the ones that are suitable for low-power WSNs. In this paper, we evaluate the error performance of RS codes with an emphasis on the optimization of design factors for RS codes to achieve better bit error performance over both Additive White Gaussian Noise (AWGN) channels and Rayleigh fading channels (Farzamnia et al., 2018).

\section{Background}

In this section, we briefly review the concept of RS codes and factors considered for evaluating the performance of RS codes, namely, channel models, modulation schemes, and performance metrics.

\subsection{RS Codes}

RS codes are the most widely used FEC codes for achieving reliable data transmissions in communication networks (Wicker and Bhargava, 1994; Shi et al., 2017). They are non-binary linear block codes that process a fixed size block of message symbols at a time. A RS $(n, k)$ code defined over GF $\left(2^{m}\right)$ with $m$-bit symbols has a block length of $n=2^{m}-1$ and $k(k<n)$ data symbols. RS codes are maximum-distance-separable (MDS) codes with code distance (i.e., the minimum Hamming distance) being $d_{\min }=n-k+1$. The RS $(n, k)$ encoder takes $k$ data symbols at a time and encodes them into $n$ coded symbols block as the codeword by adding $(n-k)$ parity symbols as redundancy. It enables a receiver to recover the $k$ data symbols from any set of exactly $k$ encoded symbols. RS codes are good at correcting both random errors and burst errors. A RS $(n, k)$ code corrects up to any combination of $t$ errors or $2 t$ erasures (given the position of a symbol error is known), where $t=(n-k) / 2$ indicates the error correction capability. $R=k / n$ is referred to as the rate of the code. Being MDS codes, RS codes make a highly efficient use of redundancy. The block lengths $n$ and characters sizes $k$ can be easily adjusted to match different channel conditions and applications needs. Therefore, RS codes are optimal in terms of the error and erasure correction capability. RS codes also provide a wide range of code rates that can be chosen such that the coded schemes have compatible bandwidth efficiency as the corresponding uncoded system. Therefore, RS codes have been studied and implemented for WSNs (Huu et al., 2012; Tan et al., 2014). In this paper, we study the impact of RS parameters $n, k, t$, and $R$ on the error performance of RS codes with the goal of optimizing their use in the WSN applications. 
International Journal of Mathematical, Engineering and Management Sciences

Vol. 5, No. 1, 13-28, 2020

https://doi.org/10.33889/IJMEMS.2020.5.1.002

\subsection{Channel Models and Modulation Schemes}

In this work, we study performance of RS codes under AWGN and Rayleigh fading channels. The AWGN channel is the most frequently assumed model for a transmission channel in the digital communication theory (Abdullah and Noori, 2011; Parthasarathy et al., 2018), the Rayleigh channel is a useful model of real-world phenomena in wireless communications (Dawa et al., 2018; Tian et al., 2018).

More specifically, an AWGN channel adds white Gaussian noises to the signal that passes through it (Shahi et al., 2018). This model does not account for fading, frequency selectivity, interference, and dispersion. Therefore, it cannot model complicated communication systems. In realistic wireless environments, multi-path fading strongly affects communications in WSNs. Multi-path fading increases the possibility of signal cancellation, which leads to a higher packet loss rate and therefore resulting in more power consumption. Thus, the wireless communication in WSNs can be more accurately modeled as a fading channel. The coding gain when using ECC codes on fading channels is generally higher than that on a Gaussian channel (Hagenauer and Lutz, 1987).

Modulation is the process of coding information onto the carrier frequency. The modulation scheme used by the radio is an important factor that strongly affects the energy consumption of the node. In this work, we study RS performance under different modulation schemes. Particularly, we choose $M$-ary Phase Shift Keying (MPSK), M-ary Frequency Shift Keying (MFSK), and Offset Quadrature Phase-Shift Keying (OQPSK) modulation schemes for the simulations of error performance of RS codes.

\subsection{Performance Metrics}

One performance metric used in the simulations is BER, which can be measured by computing bit error probability $P_{b}$. Different modulation schemes have different values for the error function, and thus have different values of $P_{b}$. Another metric used is the energy per bit to noise power spectral density ratio $\left(E_{b} / N_{o}\right)$, which is a normalized measure of SNR. It is also known as "SNR per bit" and is often used to find how much transmitter power is needed for a certain digital link. In this work, we compare the BER vs. $E_{b} / N_{o}$ performance of RS codes by varying values of RS parameters. Results are also compared with those of corresponding un-coded systems.

\section{Error Performance on AWGN Channels}

In this section, we study RS error performance for MPSK signaling over AWGN channels.

\subsection{Uncoded System}

Figure 1 shows the basic modulator/demodulator block diagram without channel coding. 
International Journal of Mathematical, Engineering and Management Sciences

Vol. 5, No. 1, 13-28, 2020

https://doi.org/10.33889/IJMEMS.2020.5.1.002

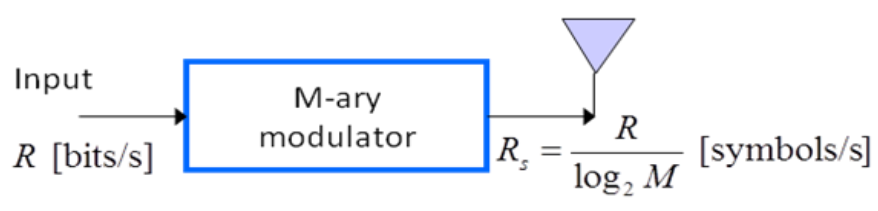

AWGN channel

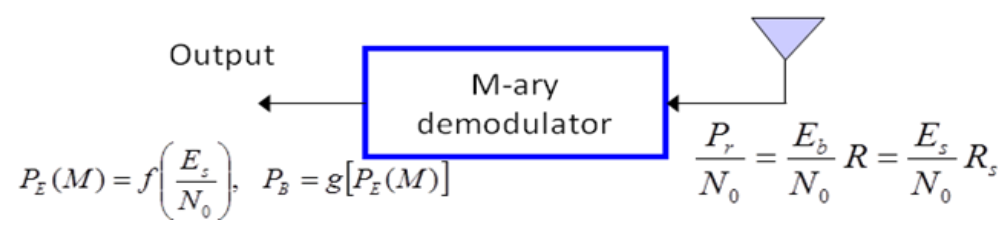

Figure 1. Basic modulator/demodulator without channel coding (Sklar, 2001)

For $M=2$ (BPSK) and $M=4$ (QPSK), the bit error probability $P_{b}$ on AWGN channel is given by (Sklar, 2001)

$$
P_{b}=Q\left(\sqrt{\frac{2 E_{b}}{N_{0}}}\right) \quad \text { or } \quad P_{b}=\frac{1}{2} \operatorname{erfc}\left(\sqrt{\frac{E_{b}}{N_{0}}}\right)
$$

The $Q$-function, $Q(x)$, is defined as $Q(x)=\frac{1}{\sqrt{2 \pi}} \int_{x}^{\infty} e^{-t^{2} / 2} d t=\frac{1}{2} \operatorname{erfc}\left(\frac{x}{\sqrt{2}}\right), x \geq 0$, where $\operatorname{erfc}(\cdot)$ is the complementary error function defined as $\operatorname{erfc}(x)=\frac{2}{\sqrt{\pi}} \int_{x}^{\infty} e^{-t^{2}} d t$.

For BPSK, $M=2, m=\log _{2}(M)=1$ (one bit per symbol), the symbol-error probability $P_{E}$ is the same as bit-error probability and can be computed using (1). The bit error probability for OQPSK is the same as for BPSK. For large $M(>4)$, the probability that the demodulator makes a symbol error $P_{E}(M)$ can be approximated by (Proakis, 2000)

$$
P_{E}(M) \approx 2 Q\left(\sqrt{\frac{2 E_{s}}{N_{0}}} \sin \left(\frac{\pi}{M}\right)\right) \text { or } P_{E}(M) \approx \operatorname{erfc}\left(\sqrt{\frac{E_{s}}{N_{0}}} \sin \left(\frac{\pi}{M}\right)\right)
$$

where $E_{s} / N_{o}$ is the ratio of energy in a symbol to noise power spectral density, and $M$ is the number of symbols, and $m=\log _{2}(M), E_{S} / N_{0}=m \cdot\left(E_{b} / N_{0}\right)$. When the Gray coding is used, $P_{b}$ can be approximated as $P_{b} \approx P_{E}(M) / \log _{2} M$. That is,

$$
P_{b} \approx \frac{2}{\log _{2}(M)} Q\left(\sqrt{2 \log _{2}(M) \cdot \frac{E_{b}}{N_{0}}} \cdot \sin \left(\frac{\pi}{M}\right)\right) \approx \frac{1}{m} \operatorname{erfc}\left(\sqrt{m \cdot r_{b}} \sin \left(\frac{\pi}{M}\right)\right)
$$

where $m=\log _{2}(M)$ is the number of bits per modulation symbol and $r_{b} \equiv E_{b} / N_{0}$ is the energy per bit to noise power density ratio. For example, for 8-PSK $(M=8, m=3)$, we have 
International Journal of Mathematical, Engineering and Management Sciences

Vol. 5, No. 1, 13-28, 2020

https://doi.org/10.33889/IJMEMS.2020.5.1.002

$$
P_{b_{8 P S K}}=\frac{1}{3} \operatorname{erfc}\left(\sqrt{3 r_{b}} \sin \left(\frac{\pi}{8}\right)\right)
$$

To determine $P_{b}$, we convert the symbol error probability $P_{E}$ to its bit error equivalent. There is no general formula for the symbol to bit error probability conversion. The actual $P_{b}$ is shown to be bounded by (Proakis, 2000)

$$
\frac{P_{E}(M)}{\log _{2} M} \leq P_{b} \leq \frac{M / 2}{M-1} P_{E}(M)
$$

The lower limit corresponds to the case where the symbols have undergone the Gray coding. The upper limit corresponds to the case of using the pure binary coding.

\subsection{RS Coded System}

Figure 2 shows the RS $(n, k)$ coded $M$-ary signaling on AWGN channel, where $R$ is data-bit rate, $R_{c}=(n / k) R$ is channel-bit rate, and $R_{s}=R_{c} /\left(\log _{2} M\right)$ is symbol rate. For a $\operatorname{RS}(n, k)$ coded system, the symbol-error probability $P_{E}$ of MPSK modulation on AWGN channels $(M>2)$ is approximated as (Odenwalder, 1976)

$$
P_{E}(M) \approx 2 Q\left(\sqrt{\frac{2 E_{s}}{N_{0}}} \sin \left(\frac{\pi}{M}\right)\right) \approx \operatorname{erfc}\left(\sqrt{\left(\log _{2} M\right) \cdot\left(\frac{k}{n}\right) \frac{E_{b}}{N_{0}}} \sin \left(\frac{\pi}{M}\right)\right)
$$

Assume hard-decision demodulation is used, the channel-bit-error probability out of the demodulator and into the decoder denoted as $P_{c}$ is expressed as $P_{c} \approx \frac{P_{E}(M)}{\log _{2}(M)}$. The final output decoded $P_{b}$ depends on the particular code, the decoder, and the channel-bit-error probability $P_{c}$. It can be approximated by (Odenwalder, 1976)

$$
P_{b} \approx \frac{1}{2^{m}-1} \sum_{j=t+1}^{2^{m}-1} j\left(\begin{array}{c}
2^{m}-1 \\
j
\end{array}\right) P_{c}^{j}\left(1-P_{c}\right)^{2^{m}-1-j}
$$

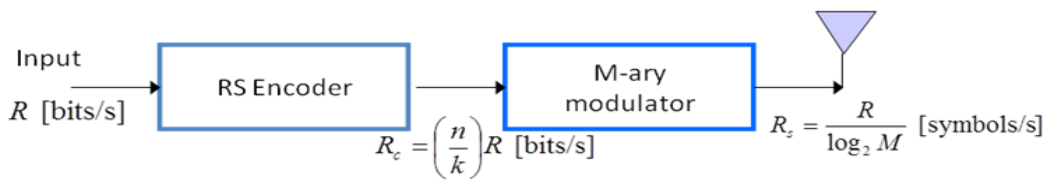

AWGN channel

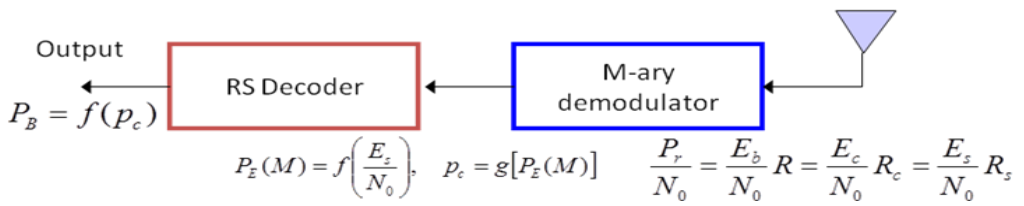

Figure 2. Basic modulator/demodulator with channel coding (Sklar, 2001) 
International Journal of Mathematical, Engineering and Management Sciences

Vol. 5, No. 1, 13-28, 2020

https://doi.org/10.33889/IJMEMS.2020.5.1.002

\section{Error Performance on Rayleigh Fading Channels}

We consider the performance of an MPSK signaling over a normalized Rayleigh fading channel as described in (Jamali and Le-Ngoc, 1991). The transmission model is depicted in Figure 3. The transmitted signal $x$ is corrupted by introducing a multipath gain represented by a complex-valued number $\underline{A}=A e^{\gamma \phi}$, and an additive Gaussian noise $\underline{N}$. The additive Gaussian noise is defined to have one-side spectral density $N_{0}$. The multiplicative gain $A$ is a random variable having a normalized Rayleigh fading density function as:

$p_{A}(a)=2 a \cdot e^{-a^{2}}$

with $E\left[A^{2}\right]=1$.

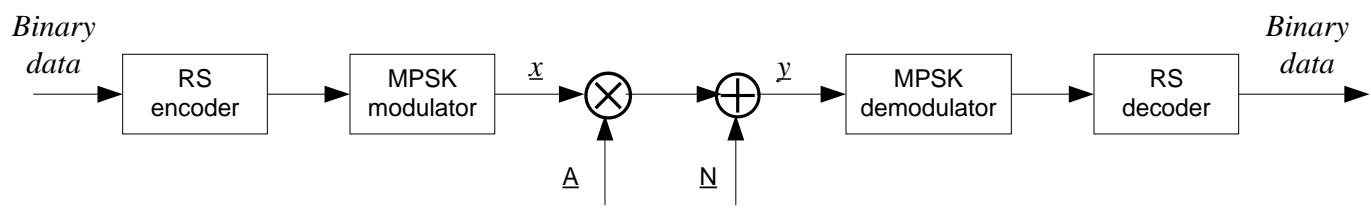

Figure 3. Signal transmission model (Jamali and Le-Ngoc, 1991)

\subsection{Uncoded System}

At high $E_{b} / N_{0}$, the symbol error probability of uncoded MPSK signals in an AWGN channel can be written approximately as (Proakis, 2000)

$$
P_{s}(a) \approx \operatorname{erfc}\left(a \sqrt{\frac{E_{s}}{N_{0}}} \sin \left(\frac{\pi}{M}\right)\right)
$$

where $a$ is channel amplitude. When $M=2^{m}$, each channel symbol includes $m=\log _{2}(M)$ channel bits and $E_{s}=m E_{b}$. After going through an encoder with coding rate $R=k / n$ being adopted, $m$ channel bits only carry $m R$ information bits. Thus, we get

$$
P_{s}(a) \approx \operatorname{erfc}\left(a \sqrt{m R \frac{E_{b}}{N_{0}}} \sin \left(\frac{\pi}{M}\right)\right)
$$

Eq. (9) can be regarded as the conditional symbol error rate given the fixed fading amplitude $a$. The symbol-error probability for MPSK signaling over a Rayleigh fading channel can be found by averaging the conditional symbol-error probability of (9) with respect to the fading amplitude $a$ :

$P_{s}=\int_{0}^{\infty} P_{s}(a) \cdot p_{A}(a) \cdot d a=1-\frac{\Gamma_{b M}}{\sqrt{1+\Gamma_{b M}^{2}}}$ 
International Journal of Mathematical, Engineering and Management Sciences

Vol. 5, No. 1, 13-28, 2020

https://doi.org/10.33889/IJMEMS.2020.5.1.002

where $\gamma_{b} \equiv E_{b} / N_{0}$, and $\Gamma_{b M} \equiv \sqrt{m R_{c} \gamma_{b}} \sin \left(\frac{\pi}{M}\right)$. For the BPSK modulation,

$P_{s}=1-\sqrt{\frac{R \gamma_{b}}{1+R \gamma_{b}}}$

The relationship between $P_{s}$ and $P_{b}$ of MPSK signals depends upon the mapping of the $m$-bit groups to the symbols. If the Gray coding is used, $P_{b}$ can be approximated by (Proakis, 2000):

$P_{b}=\frac{P_{s}}{\log _{2}(M)}=\frac{P_{s}}{m}$

\subsection{RS Coded System}

Consider a RS $(n, k)$ code defined over $G F\left(2^{m}\right)$ with error-correction capability of $t=\lfloor(n-k) / 2\rfloor$.

If the number of errors in the received codeword exceeds the error correction capability of the code, the decoding failure occurs (i.e., the decoder cannot recover the correct data word). The upper bound of the post decoding bit-error probability for the erasure-and-error decoding of the RS coded system is given as (Clark Jr. and Cain, 1981; Zhang et al., 2000).

$P_{b} \leq \frac{2^{m-1}}{2^{m}-1} \sum_{j=t+1}^{2^{m}-1} \frac{j+t}{2^{m}-1}\left(\begin{array}{c}2^{m}-1 \\ j\end{array}\right) p_{c}^{j}\left(1-p_{c}\right)^{2^{m}-1-j}$

where $P_{c}$ is the code symbol error rate of the RS code. For the first approach of the RS coded scheme (RS over $G F\left(2^{m}\right)+2^{m}$-PSK), where each code symbol is mapped directly to one channel symbol, $P_{c}=P_{s}$. For the second approach (RS over $G F\left(2^{l m}\right)+2^{m}$-PSK), where each code symbol is composed of $l$ concatenated MPSK symbols, the probability that each code symbol is correctly received is $\left(1-P_{s}\right)^{l}$ and the code symbol error rate is

$P_{c}=1-\left(1-P_{s}\right)^{l}$

\section{Error Performance Comparison}

Figure 4 shows the error performance of an example RS $(7,5)$ coded 8-PSK system over the AWGN channel and Rayleigh channel, as compared to the corresponding uncoded system. It can be seen from the figure that the error performance of the transmitted signal is greatly improved by using the RS code for both AWGN channel and Rayleigh channel, and the RS coded system has more coding gains in the Rayleigh channel than in the AWGN channel. 
International Journal of Mathematical, Engineering and Management Sciences

Vol. 5, No. 1, 13-28, 2020

https://doi.org/10.33889/IJMEMS.2020.5.1.002

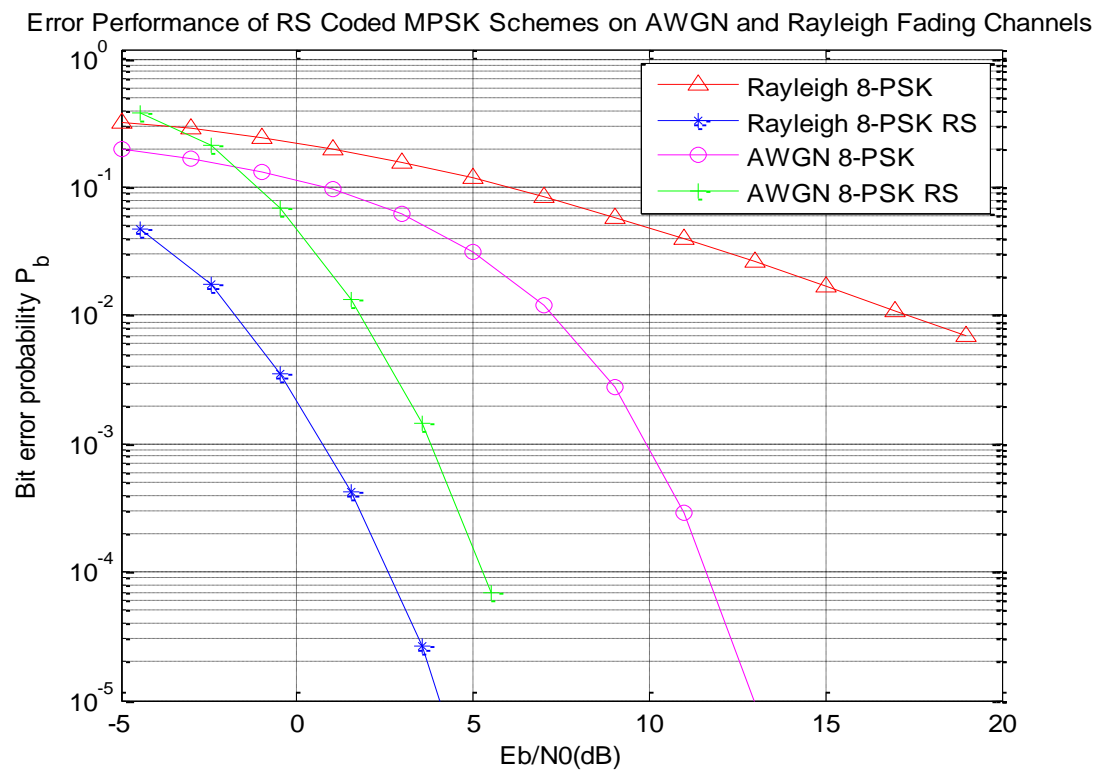

Figure 4. Error performance of RS coded MPSK schemes

\section{Effects of RS Design Parameters}

In this section, we evaluate RS error performance as a function of the RS code parameters, namely, $n, k, R$, and $t$. Effects of different modulation schemes on the BER performace of the system are also investigated.

\subsection{Constant $n$, Increasing $t$}

Figures 5 and 6 show the BER respectively under the 32-ary FSK and OQPSK modulations over an AWGN channel with constant $n=31$ and increasing error correcting capabilities $t$ ranging from 1 to 8 . It can be seen that, with constant block length $n$, the BER performance improves with the increase of $t$ from 1 to 5 , it degrades when $t>5$. The reason is with constant $n$, increasing $t=$ $(n-k) / 2$ means decreasing $k$ thus the code rate $R=k / n$. It also shows that RS $(31,21)$ with $t=5$ and $R=0.68$ is the optimum code in this case, and it has a code gain of about $3.3 \mathrm{~dB}$. The degradation in the curve is because the amount of processing "power" required for RS encoding and decoding is related to the number of parity symbols per codeword. A large value of $t$ means that a large number of errors can be corrected but requires more computational power than a small value of $t$.

The specific observations are 1) As $E_{b} / N_{0}$ increases, i.e., the channel condition improves, the BER performance improves; 2) When $E_{b} / N_{o}$ is small, i.e., the channel condition is bad, the BER performance using RS code is worse than that without using RS code because the number of occurrence of erroneous bits can exceed the error correction capability of RS code; and 3) There are $E_{b} / N_{o}$ degradations for very large code rates (less redundancy) and very low code rates (large redundancy) compared to the optimum rate: as the code rate approaches unity (no coding), the system will suffer worse error performance; at very low code rates, the more energy reduction per bit, more errors the demodulator makes, the worse the BER performance. The above observations are also applicable to simulation cases discussed in the following subsections. 
International Journal of Mathematical, Engineering and Management Sciences

Vol. 5, No. 1, 13-28, 2020

https://doi.org/10.33889/IJMEMS.2020.5.1.002

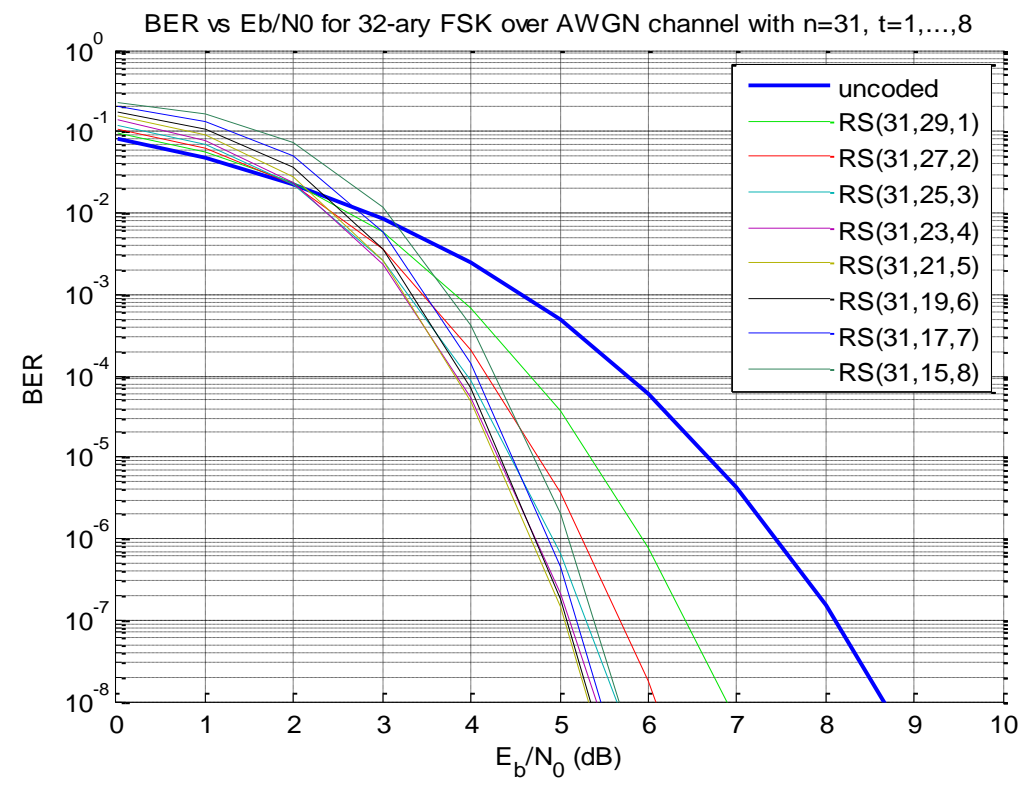

Figure 5. RS error performance for MFSK over AWGN channel with constant $n$ and increasing $t$

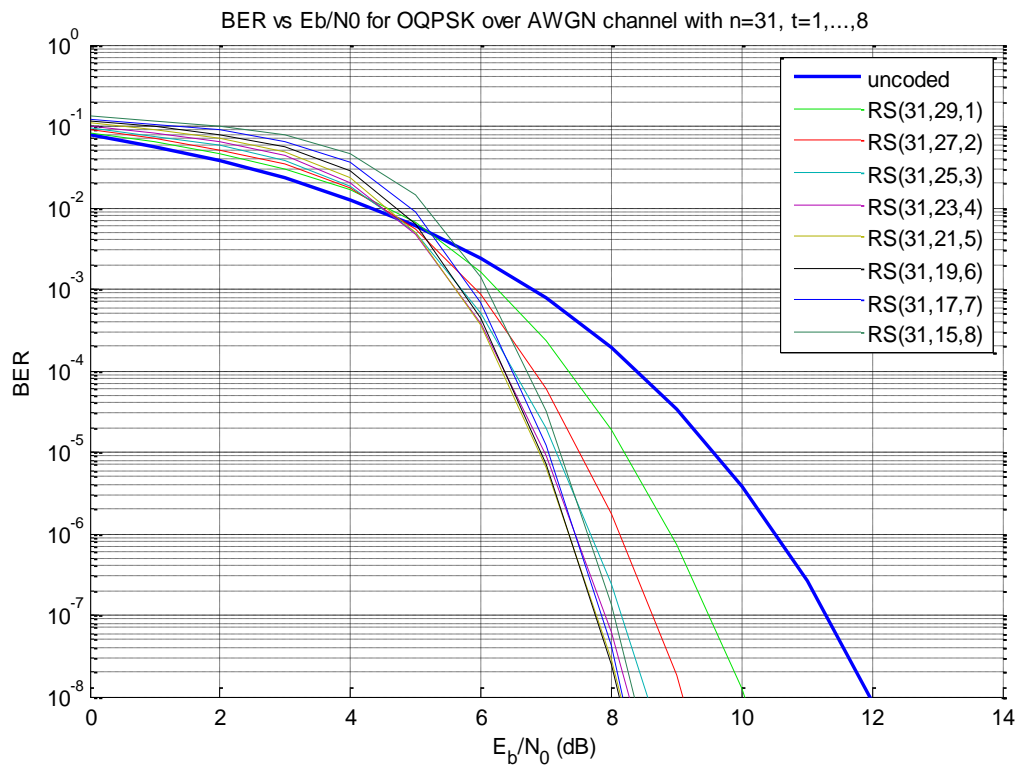

Figure 6. RS error performance for OQPSK over AWGN channel with constant $n$ and increasing $t$

\subsection{Constant $R$, Increasing $n$}

Keeping constant code rate $R$, the error performance of RS improves and the error correction capability $t$ increases as the code block size $n$ increases, making RS codes an attractive choice 
International Journal of Mathematical, Engineering and Management Sciences

Vol. 5, No. 1, 13-28, 2020

https://doi.org/10.33889/IJMEMS.2020.5.1.002

whenever long block lengths are desired. This can be seen in Figures 7 and 8, where the code rate is held at a constant 0.875 , while its block size increases from $n=15$ symbols (with $m=4$ bits per symbol) to $n=255$ symbols (with $m=8$ bit per symbol).

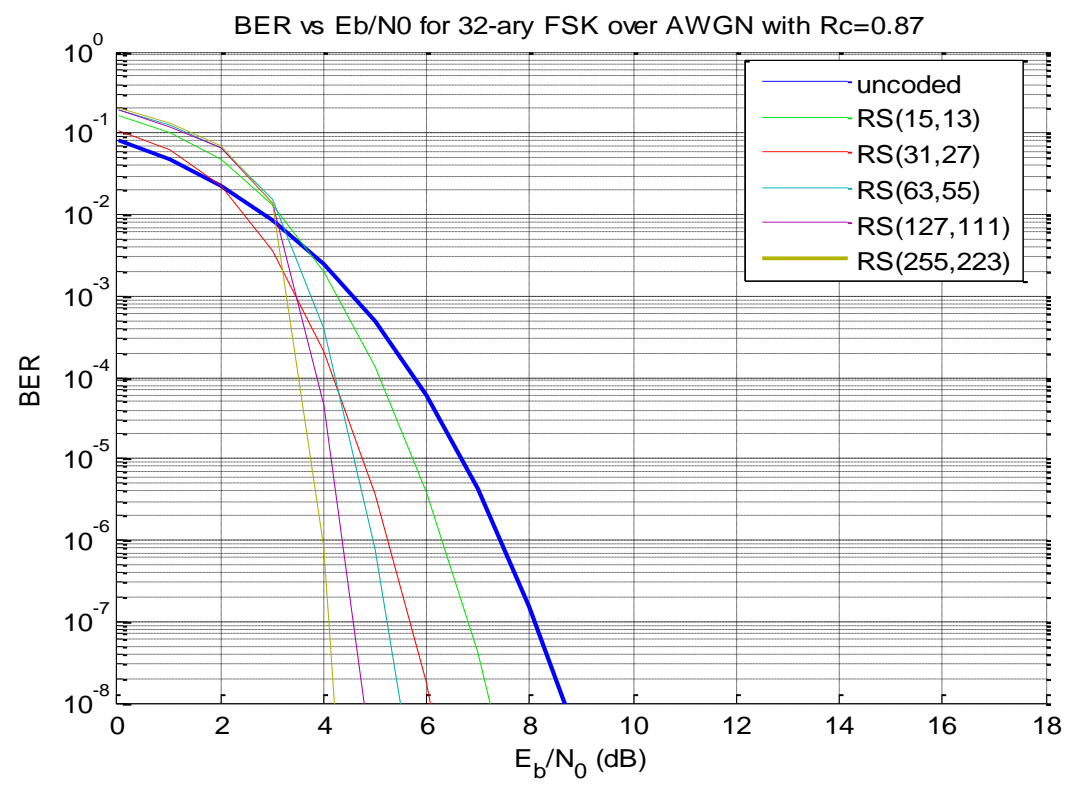

Figure 7. RS error performance for MFSK over AWGN channel with constant $R$ and increasing $n$

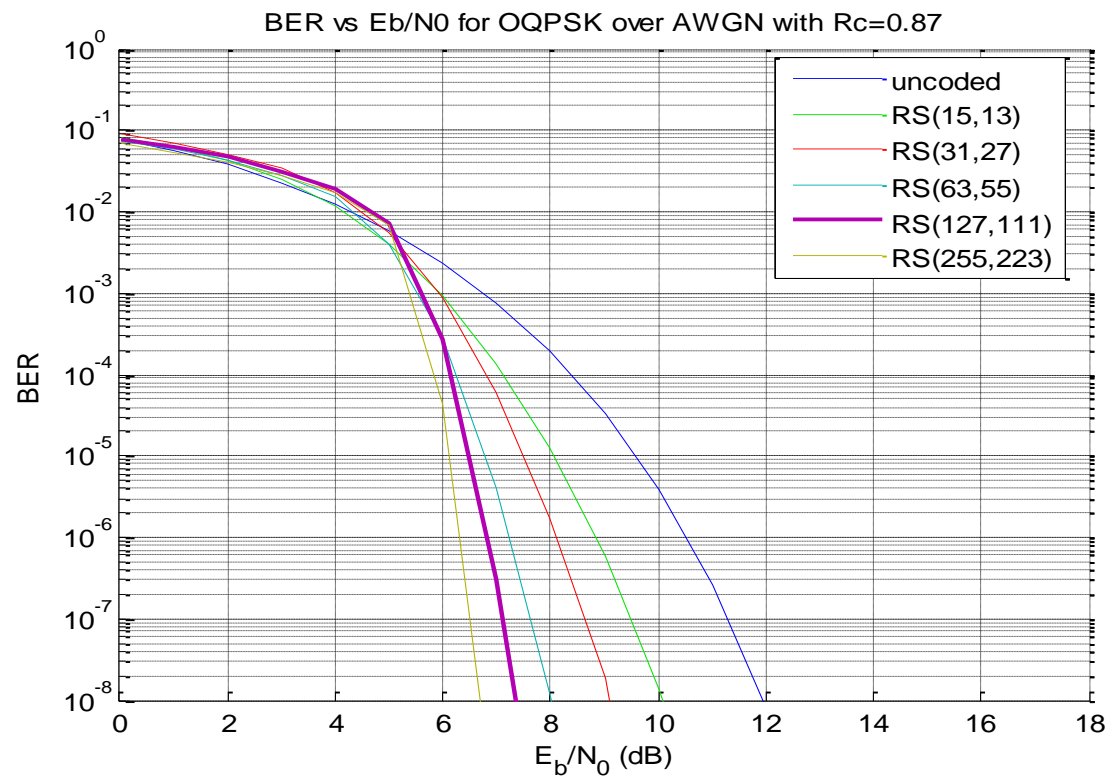

Figure 8. RS error performance for OQPSK over AWGN channel with constant $R$ and increasing $n$ 
International Journal of Mathematical, Engineering and Management Sciences

Vol. 5, No. 1, 13-28, 2020

https://doi.org/10.33889/IJMEMS.2020.5.1.002

\subsection{Constant $k$, Increasing $\boldsymbol{n}-\boldsymbol{k}$}

With constant $k$, an increase in redundancy $n-k$ means the increase of codeword length $n$ and error correction capability $t$ but a decrease in $R$; the larger codes rate result in better BER performance, as shown in Figures 9 and 10.

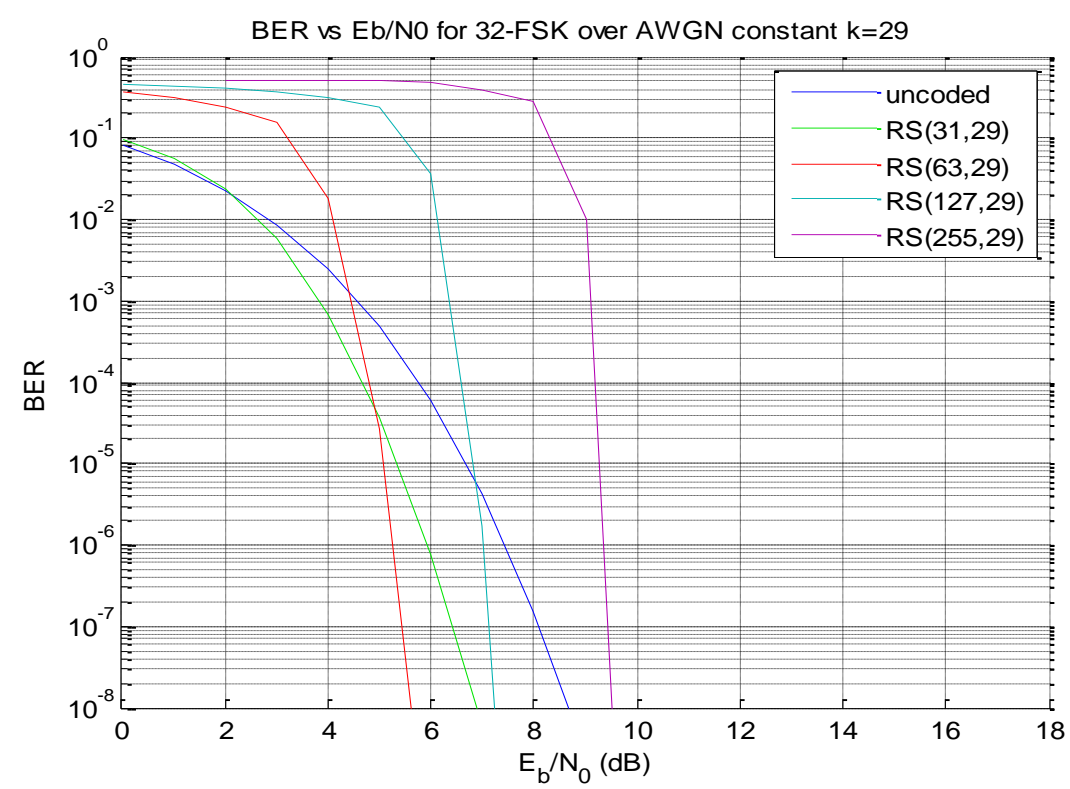

Figure 9. RS error performance for MFSK over AWGN channel with constant $k$, increasing $n-k$

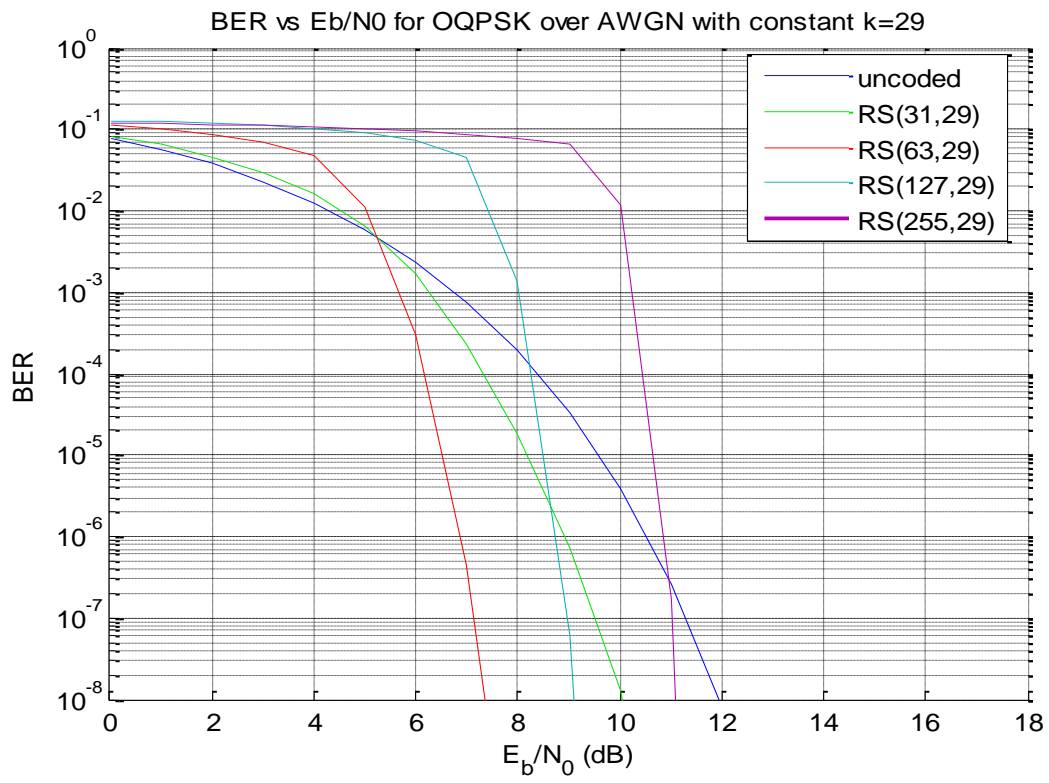

Figure 10. RS error performance for OQPSK over AWGN channel with constant $k$, increasing $n-k$ 
International Journal of Mathematical, Engineering and Management Sciences

Vol. 5, No. 1, 13-28, 2020

https://doi.org/10.33889/IJMEMS.2020.5.1.002

\subsection{Constant $t$, Increasing $n$}

In this case, the optimum code rate for a RS code is calculated from the decoder performance at various code rates. The code rate which requires the lowest $E_{b} / N_{0}$ for a given BER is chosen as the optimum code rate for RS code design. In the simulations, we keep $t$ as a constant with values of 2 and 5 respectively and increase code length $n$ from 15 to 255 for OQPSK modulation over an AWGN channel. Figures 11 and 12 show that with constant $t$, RS error performance improves as the increase of $n$ from 15 to 255. By comparing results in Figures 11 and 12, we can see that increasing $t$ improves the RS error performance. The optimum code rate is between 0.67 and 0.87 .

We compare RS error performance for different modulation schemes on AWGN channels. The RS code used in this simulation is RS $(31,21)$ which is shown to be an optimum RS code in the previous simulations. Figure 13 shows that among all the tested modulation schemes, 32-FSK has the best error performance while 16-QAM has the worst error performance. The RS coding gain of all the modulation schemes is about $4 \mathrm{~dB}$.

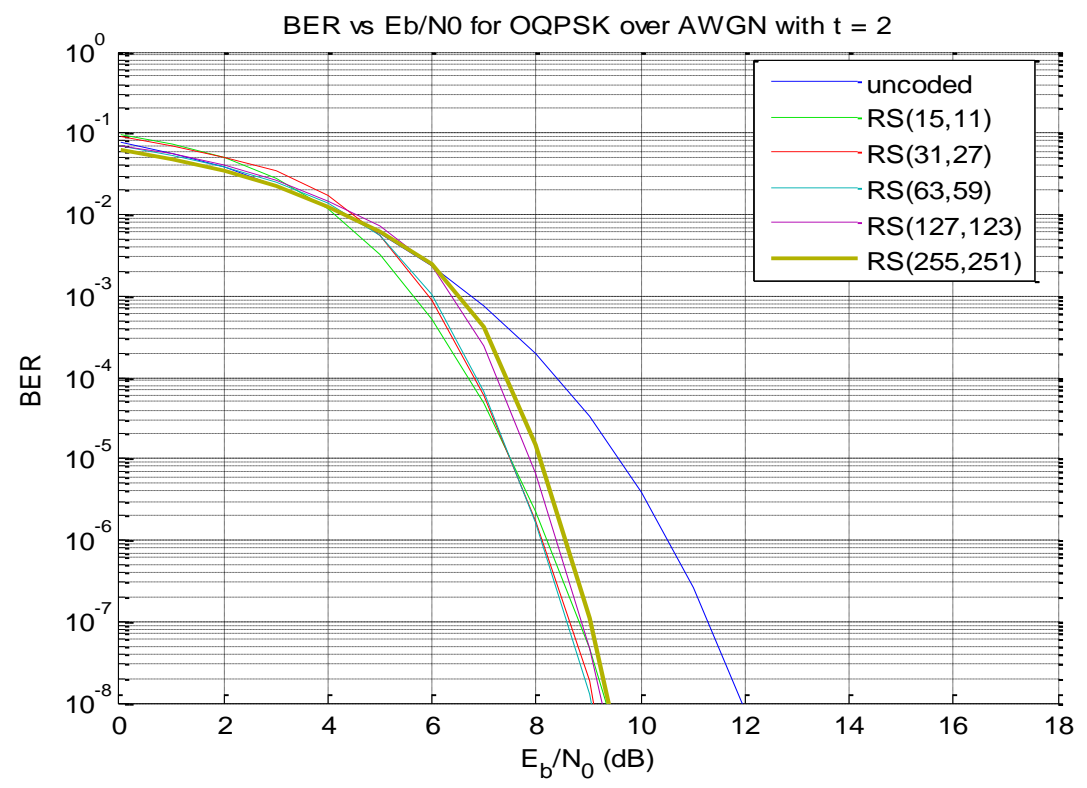

Figure 11. RS error performance for OQPSK over AWGN channel with $t=2$ and increasing $n$ 
International Journal of Mathematical, Engineering and Management Sciences

Vol. 5, No. 1, 13-28, 2020

https://doi.org/10.33889/IJMEMS.2020.5.1.002

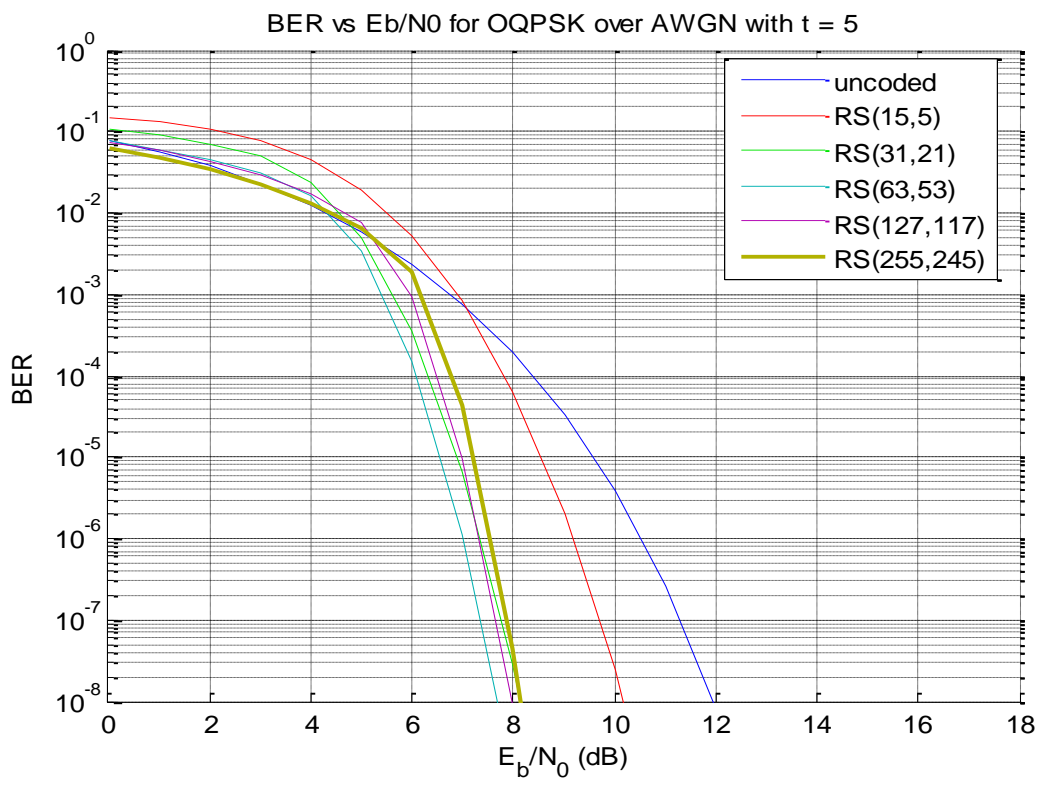

Figure 12. RS error performance for OQPSK over AWGN channel with $t=5$ and increasing $n$

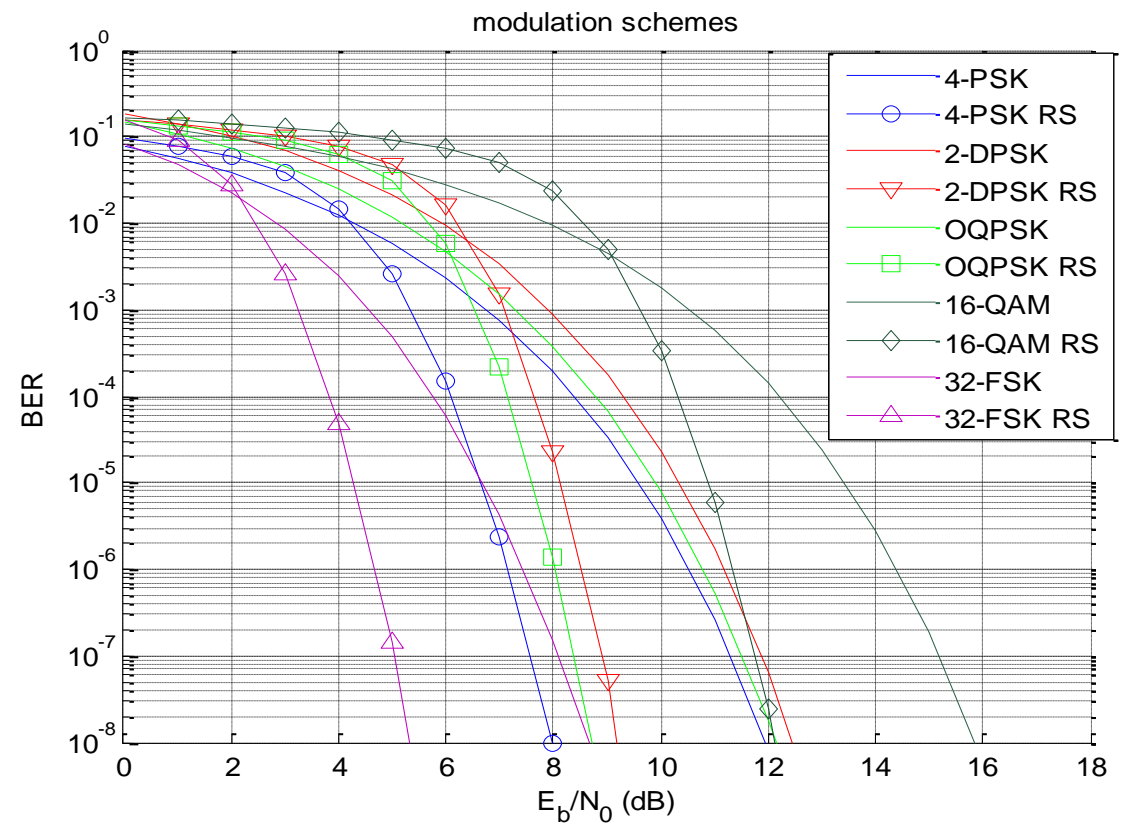

Figure 13. RS error performance for difference modulation schemes

\section{Conclusions}

In this paper, we present formulas for evaluating the error performance of RS codes (BER vs. $\left.E_{b} / N_{0}\right)$ for both AWGN and Rayleigh fading channels. Effects of different design factors of RS 
International Journal of Mathematical, Engineering and Management Sciences

Vol. 5, No. 1, 13-28, 2020

https://doi.org/10.33889/IJMEMS.2020.5.1.002

codes and different modulation schemes have been investigated. The simulation results show that using RS coded modulation schemes provides better reliability performance for the transmitted signals than uncoded systems for both AWGN and Rayleigh fading channels. With the proper design of RS code parameters and choice of modulation schemes, significant coding gains can be obtained by using RS codes. Particularly, among all the studied modulation schemes, the MFSK modulation performs the best while MQAM performs the worst. Simulation results also show that the BER performance is initially improved as code rate $R$ increases, but it eventually decreases after passing a certain threshold value of $R$. The optimum code rate is between 0.67 and 0.87 . With constant $R$, the BER performance improves as the codeword length $n$ increases.

\section{Conflict of Interest}

The authors confirm that there is no conflict of interest to declare for this publication.

\section{Acknowledgments}

This research did not receive any specific grant from funding agencies in the public, commercial, or not-for-profit sectors. The authors sincerely appreciate the editor and reviewers for their time and valuable comments.

\section{References}

Abdullah, H.N., \& Noori, A.M. (2011). Performance evaluation of selected serial concatenation codes in AWGN \& frequency selective Rayleigh fading channels. Journal of Engineering and Sustainable Development, 15(1), 118-125.

Abedi, A. (2011). Power-efficient-coded architecture for distributed wireless sensing. IET Wireless Sensor Systems, 1(3), 129-136.

Alrajeh, N.A., Marwat, U., Shams, B., \& Shah, S.S.H. (2015). Error correcting codes in wireless sensor networks: an energy perspective. Applied Mathematics \& Information Sciences, An International Journal, 9(2), 809-818.

Bettayeb, M., Ghunaim, S., Mohamed, N., \& Nasir, Q. (2019). Error correction codes in wireless sensor networks: a systematic literature review. In 2019 International Conference on Communications, Signal Processing, and their Applications (ICCSPA) (pp. 1-6). IEEE. Sharjah, United Arab Emirates.

Clark Jr, G.C., \& Cain, J.B. (1981). Error-correction coding for digital communications. Applications of Communications Theory. Plenum Press, New York, London.

Dawa, M., Kaddoum, G., \& Sattar, Z. (2018). A generalized lower bound on the bit error rate of DCSK systems over multi-path Rayleigh fading channels. IEEE Transactions on Circuits and Systems II: Express Briefs, 65(3), 321-325.

Farzamnia, A., Hlaing, N.W., Mariappan, M., \& Haldar, M.K. (2018, August). BER comparison of OFDM with M-QAM modulation scheme of AWGN and Rayleigh fading channels. In 2018 9th IEEE Control and System Graduate Research Colloquium (ICSGRC), (pp. 54-58). IEEE. Shah Alam, Malaysia.

Hagenauer, J., \& Lutz, E. (1987). Forward error correction coding for fading compensation in mobile satellite channels. IEEE Journal on Selected Areas in Communications, 5(2), 215-225.

Howard, S.L., Schlegel, C., \& Iniewski, K. (2006). Error control coding in low-power wireless sensor networks: when is ecc energy-efficient? EURASIP Journal on Wireless Communications and Networking, 2006(74812), 1-14. 
International Journal of Mathematical, Engineering and Management Sciences

Vol. 5, No. 1, 13-28, 2020

https://doi.org/10.33889/IJMEMS.2020.5.1.002

Huu, P.N., Tran-Quang, V., \& Miyoshi, T. (2012, August). Multi-hop Reed-Solomon encoding scheme for image transmission on wireless sensor networks. In $20124^{\text {th }}$ International Conference on Communications \& Electronics (ICCE), (pp. 74-79). IEEE. Hue, Vietnam.

Islam, M.R., \& Han, Y.S. (2011). Cooperative MIMO communication at wireless sensor network: an error correcting code approach. Sensors, 11(10), 9887-9903.

Jamali, S.H., \& Le-Ngoc, T. (1991, June). On the performance of bandwidth efficient RS coded MPSK signaling over a rayleigh fading channel. In ICC 91 International Conference on Communications Conference Record, (pp. 1090-1094). IEEE. Denver, CO, USA.

Ma, R., Xing, L., \& Michel, H.E. (2006, September). Fault-intrusion tolerant techniques in wireless sensor networks. In 2006 2nd IEEE International Symposium on Dependable, Autonomic and Secure Computing (DASC'06), (pp. 85-94). IEEE. Indianapolis, IN, USA.

Ma, R., Xing, L., \& Michel, H.E. (2007, May). A new mechanism for achieving secure and reliable data transmission in wireless sensor networks. In 2007 IEEE Conference on Technologies for Homeland Security (HST), (pp. 274-279). IEEE. Woburn, MA, USA.

Ma, R., Xing, L., Jin, T., \& Song, T. (2009, July). A data transmission mechanism for survivable sensor networks. In 2009 IEEE International Conference on Networking, Architecture, and Storage (NAS), (pp. 9-15), IEEE. Hunan, China.

Ma, R., Xing, L., Michel, H.E., \& Vokkarane, V.M. (2012, November). Linear cryptanalysis of a survivable data transmission mechanism for sensor networks. In 2012 IEEE Conference on Technologies for Homeland Security (HST), (pp. 562-567). IEEE. Waltham, MA, USA.

Ma, R., Xing, L., Michel, H.E., \& Wang, H. (2011). Survivable data transmission via selective hybrid cipher in sensor networks. International Journal of Performability Engineering, 7(4), 303-312.

Odenwalder, J.P. (1976). Error control coding handbook. Linkabit Corporation, San Diego, CA.

Parthasarathy, S., Kumar, S., Ganti, R.K., Kalyani, S., \& Giridhar, K. (2018). Error vector magnitude analysis in generalized fading with co-channel interference. IEEE Transactions on Communications, 66(1), 345-354.

Proakis, J.G. (2000). Digital Communications. $4^{\text {th }}$ Edition, McGraw-Hill, New York.

Shahi, S., Tuninetti, D., \& Devroye, N. (2018). On the capacity of the AWGN channel with additive radar interference. IEEE Transactions on Communications, 66(2), 629-643.

Shi, L., Jiao, J., Sabbagh, A., Wang, R., Yu, Q., Hu, J., Wang, H., Burleigh, S.C., \& Zhao, K. (2017). Integration of Reed-Solomon codes to Licklider transmission protocol (LTP) for space DTN. IEEE Aerospace and Electronic Systems Magazine, 32(4), 48-55.

Sklar, B. (2001). Digital communications: fundamentals and applications. $2^{\text {nd }}$ Edition, Prentice-Hall, Upper Saddle River, NJ.

Tan, P.L., Cheah, C.L., \& Ho, C.K. (2014, July). Hardware implementation of Reed-Solomon error correction technique for wireless sensor network based on error pattern analysis. In 2014 IEEE Region 10 Symposium, (pp. 347-350). IEEE. Kuala Lumpur, Malaysia.

Tian, L., Zhong, J., Zhao, M., \& Wen, L. (2018, June). A suboptimal algorithm for SCMA codebook design over uplink Rayleigh fading channels. In 2018 IEEE 87th Vehicular Technology Conference (VTC Spring), (pp. 1-5). IEEE. Porto, Portugal.

Wang, C., Xing, L., Zonouz, A.E., Vokkarane, V.M., \& Sun, Y. (2017). Communication reliability analysis of wireless sensor networks using phased-mission model. Quality and Reliability Engineering International, 33(4), 823-837. 
International Journal of Mathematical, Engineering and Management Sciences

Vol. 5, No. 1, 13-28, 2020

https://doi.org/10.33889/IJMEMS.2020.5.1.002

Wicker, S.B., \& Bhargava, V.K. (1994). Reed-Solomon codes and their applications. IEEE Press, Piscataway, NJ.

Yigit, M., Boluk, P.S., \& Gungor, V.C. (2019). A new efficient error control algorithm for wireless sensor networks in smart grid. Computer Standards \& Interfaces, 63, 27-42.

Zhang, L., Cao, Z., \& Gao, C. (2000, September). Application of RS-coded MPSK modulation scenarios to compressed image communication in mobile fading channel. In $52^{\text {nd }}$ Vehicular Technology Conference Fall 2000, IEEE VTS-Fall VTC 2000, (Vol. 3, pp. 1198-1203). IEEE. Boston, MA, USA.

Zonouz, A.E., Xing, Vokkarane, V.M., \& Sun, Y. (2015). Application communication reliability of wireless sensor networks. IET Wireless Sensor Systems, 5(2), 58-67. 